\title{
Dissociation, Association, or Addition? Conceptualizing the Interplay of Mechanisms for Recombinant Digital Innovation in Service Systems
}

\author{
Hubertus M. L. Waltermann \\ LMU Munich \\ waltermann@bwl.lmu.de
}

\begin{abstract}
Recombinant innovation is a useful approach to generate new digital service solutions with the help of established resources. However, it is unclear how its underlying mechanisms are interrelated and why they are applied. Based on theory from service systems and digital innovation, we analyze the interplay of dissociative, associative, and additive recombination by using the example of the media industry in a multiple case study. We find that the three mechanisms are applied in a supplementary concatenation and triggered by internal and external drivers. We contribute to IS literature by developing a conceptual model that illustrates the nexus of the mechanisms based on digital technologies as the central resource in service systems.
\end{abstract}

\section{Introduction}

Due to the outbreak of Covid-19, stores, offices and other facilities had to be locked down. Therefore, many organizations had to stop offering their established analogous services. Consequently, they reacted by quickly developing new digital service solutions that were useable under the changed circumstances of the pandemic and thereby created new value propositions. By adapting and expanding their established service systems with the help of digital technologies (DigTech), many organizations were able to offer such recombined solutions to their customers. This frequently applied approach can be referred to as recombinant digital innovation, which describes the process of reconfiguring established resources to create new interrelated services $[1,2]$.

Being a central concept in innovation research from IS $[3,4]$, the theoretical foundations of recombinant innovation are substantial [e.g. 5, 6, 7] and applied in diverse fields, such as cloud computing [8] and knowledge recombination [9]. Organizations apply recombination by dissociating, associating, and adding digital resources in their services systems. These three practices can be described as mechanisms of recombinant innovation, which can be applied to create new service solutions [10]. Interestingly, empirical insights on recombination are rather scarce [11] and if available they tend to be highly specific. For example, recent research investigated how innovative services are developed through recombination for car driver information systems [12], or how the preceding processes of recombination of services can be conceptualized for the construction industry [13]. What these studies have in common is that on the one hand they rather focus on very specific application areas. On the other hand, they consider the processes of recombination rather as a homogenous approach than as a set of distinct mechanisms. Consequently, it is important to analyze the nexus of the recombinant mechanisms of DigTech to help organizations to decide between appropriate paths for the generation of recombined digital service solutions within their service systems. Such insights would highlight the importance of a modularized technical service structure, which enhances the effectiveness of innovation processes in service systems.

To the best of our knowledge, the concatenation of dissociation, association, and addition, as well as factors triggering their individual application have not yet been fully examined. This is an issue because organizations face several challenges regarding the creation of new value propositions within already established service systems [10]. Consequently, two research gaps can be identified: First, research about the interplay of the three recombination mechanisms is missing. Second, it is unclear, why organizations decide for which mechanism within their service systems. Therefore, detailed insights on the applicability of recombination mechanisms are needed [14]. Hence, the following two research questions can be proposed:

RQ1: How can the interplay of mechanisms for recombinant digital innovation in service systems be described?

RQ2: Which drivers trigger the application of each of the three mechanisms of recombinant digital innovation in service systems? 
To answer both questions, we conducted an explorative, qualitative-empirical multiple case study, which analyzes three media organizations that use recombinant digital innovation to generate new service solutions. We decided on this industry for two reasons. At first, by being a forerunner in the development of digital services [15], the media industry is particularly affected by recombinant approaches. Secondly, the diffusion of mobile devices and constant advancements in internet speed and availability enables users to consume media contents at any time and place and therefore requires constant development of new service solutions [16].

Our results indicate that association mechanisms are preceded by the dissociation of established service solutions into recombinable digital modules, while addition mechanisms recombine new DigTech with established digital service solutions to generate new recombined digital service solutions. Moreover, dissociation is rather facilitated by external impulses, while association and addition are applied because of restrictive internal impulses. Our study contributes by deepening the understanding of the interaction of recombination mechanisms in service systems [e.g. 3, 5, $10,11,17]$. Furthermore, we shed light on the influence of external and internal drivers on the application of these mechanisms. We summarize our findings in three propositions and illustrate them by developing a conceptual model of the concatenation of mechanisms of recombinant digital innovation in service systems.

\section{Conceptual background}

For the conceptual background of our study, we first outline how the recombination of DigTech can be embedded as an innovation approach within service systems. Afterward, we describe the three mechanisms of recombinant innovation and how they are capable of generating new recombined digital service solutions. Both concepts together serve as a theoretical framework to analyze the three cases.

\subsection{Recombinant digital innovation in service systems}

In service science, service systems can be seen as a theoretical construct that helps to understand how configurations of specific resources jointly lead to the co-creation of value. These resources can have manifold forms, such as people, shared information, other service systems, and (digital) technologies [18]. For the context of digital innovation from an organizational perspective, we therefore focus on technologies as the main resources within a service system. Consequently, the outcome of activities that reconfigure such resources can be seen as the generation of service innovations that provide value propositions which are transformed into actual additional value through the users that consume these services [19]. Based on this conceptualization, an innovative digital service solution can be seen as the actual artifact (i.e. service) that is offered to users [7, 20]. From a digital innovation perspective, this means that the resources of a service system are DigTech, which are used for the generation of new digital service solutions. Hence, for this study, we define a service system as a combination of diverse DigTech that jointly form digital service solutions.

Against this background, such solutions can be generated through various innovation approaches of which one is recombination. Generally speaking, this concept describes the idea that "novel products and services derive from the carrying out of new combinations of components" [5, p. 89]. This means that the focus of this type of innovation rather lies on how than which resources are being combined [1]. The reconfiguration of DigTech within a service system can therefore be described by the concept of design recombination [11]. This concept is especially applicable to DigTech as they enhance modularity and compatibility in a service system because of their three unique properties [6]: Firstly, DigTech are reprogrammable which means that their functions can be changed dynamically. Hence, they can be aligned easily to new contexts, which in our case are incorporated by other service solutions. This means that a resource that was originally implemented in a digital solution within a service system can easily be adapted and implemented into another solution within this system. Secondly, DigTech are data-homogenous which enables communication between all DigTech within a service system. This means that they can be decoupled from their original service solution to be combined with other DigTech to generate a new (recombined) innovative digital service solution. Thirdly, DigTech provide a self-referential nature. This means that their utilization within a specific service system normally requires the use of other DigTech. This leads to the emergence of positive network effects which enhance the application of recombination [7].

These three properties allow that previously uncombined resources (i.e. DigTech) can set up new relationships with each other, which in return leads to the generation of recombined digital service solutions [21]. Such solutions must meet two assumptions: On the one hand, DigTech that are connected to build a recombined digital service solution must be delimited from each other. This means that each technology within a recombined solution must be independent of its original configuration, as well as from other 
technologies [10]. On the other hand, a solution must consist of at least one resource that is already established within another digital solution to be accountable for being recombined. Consequently, the more a solution consists of resources that are already used in configurations of other established solutions, the more it can be seen as an outcome of recombinant innovation [2]. A suitable example for such a recombined digital service solution is a streaming service: In essence, its value stems from the novelty of the way its resources are recombined and not from the novelty of the resources themselves (e.g. digitally stored contents offered via a website interface). Against this background, we define recombinant digital innovation in service systems as a specific type of service engineering that has recombined digital service solutions as an outcome.

\subsection{Mechanisms and drivers of recombinant digital innovation in service systems}

Following Beverungen et al. [10] recombination processes in service systems follow three distinct mechanisms: At first, dissociation mechanisms aim at splitting up an established service solution into its resources to use them individually and apart from each other as new recombined service solutions. Secondly, association mechanisms seek for setting up new connections between resources of already established solutions. By generating such new ties between the resources of two or more services, a new recombined service solution emerges. Thirdly, addition mechanisms comprise the supplementation of an established solution with a new resource, which ultimately leads to the generation of new recombined service solutions. Dissociative, associative, and additive recombination mechanisms are central to the design of service systems [22]. Against this background, we define the mechanisms of recombinant digital innovation in service systems as engineering patterns that reconfigure the resources (i.e. DigTech) of established digital services to create new recombined digital services that provide new value propositions.

Although Beverungen et al. [10] propose that recombinant innovation can be triggered through resources from internal and external sources, the actual reasons for the application for one of the three mechanisms remain unclear. At this point, the work of Lundberg et al. [13] provides first empirical insights: They find that three conditions, namely firm misalignment, environmental change, and innovation exploration serve as triggers for the recognition of opportunities for recombination. These triggers lead to the emergence of new paths for value creation and therefore enable resource recombination. Hence, it can be implied that a set of diverse factors can lead to the application of recombinant mechanisms in an organization. We define these factors as drivers for recombination in service systems.

In essence, on the one hand, it is unclear if the three mechanisms operate separately from each other and therefore mutually exclusive or if there is a concatenation between dissociation, association, and addition. On the other hand, the drivers that trigger the application of one of the recombination mechanisms are ambiguous. Hence, the theoretical foundation for the conceptual framework of our case study implies that established digital service solutions are composed of DigTech established within a service system. Due to diverse drivers, recombined digital service solutions are generated through dissociation, association, and addition of these DigTech.

In Figure 1, we illustrate our conceptual framework that summarizes the theoretical foundation of our study: Within a service system, established digital service solutions exist. Through dissociation, association, and addition of the resources (i.e. DigTech) of these solutions, recombined digital service solutions can be generated. The application of one of the three mechanisms is dependent on diverse drivers. This framework serves as a central theoretical lens for the analysis of our case data, which will be further outlined in the next section.

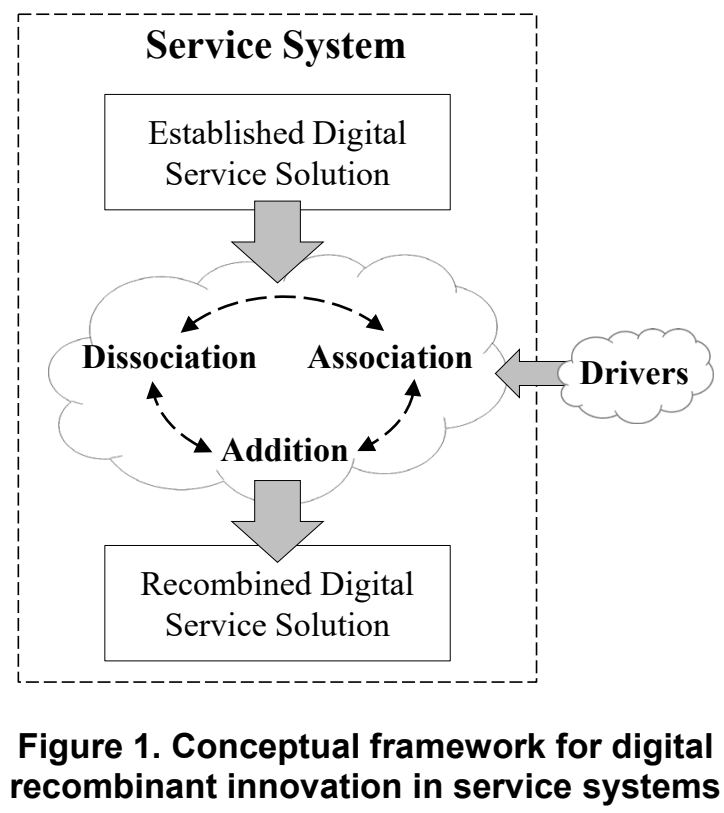

\section{Research Approach}

As outlined before, empirical research on the interplay between the mechanisms of recombinant digital innovation and its drivers in service systems is scarce. Therefore, we conducted three case studies, 
which are a suitable instrument to study phenomena within their real-life context when insights about this topic are tenuous [23]. Hence, we decided to follow an explorative positivist, multiple case study research design to analyze objectively how the three mechanisms of recombinant innovation are applied in organizations. Based on our findings we derived propositions that can serve as a basis for further research [24]. To strengthen the scientific rigor of our empirical study and to enhance the quality of our positivist multiple case study, we followed established scientific guidelines and recommendations for qualitative research $[25,26]$.

\subsection{Case selection and sample description}

Following Vargo \& Lusch, services can be defined as " $[\ldots]$ the application of specialized competencies (knowledge and skills) through deeds, processes, and performances for the benefit of another entity or the entity itself" [27, p. 1]. Against this background, the cases for our study must apply internal competencies to generate new value for users in terms of recombined digital service solutions. A reasonable example for such organizations are media companies, which offer services to their customers by creating, editing, aggregating, and providing contents [28]. Especially because of the pervasive digitalization [14], media companies applied diverse recombination mechanisms to reutilize the DigTech that transfer their contents and thereby generate new innovative service solutions [22].

Hence, in accordance with Benbasat et al. [29], the media industry is a suitable setting for this case study due to the following factors: Firstly, the object of investigation (i.e. application of recombination mechanisms in digital service systems) cannot be studied outside of its natural setting. Secondly, the digital transformation of media services is a contemporary development. Thirdly, we do not involve experimental controls or manipulations in our research. Lastly, although there is a stable theoretical basis for digital innovations and recombination mechanisms, there are no theoretical insights on the consolidation of these two concepts with a focus on service systems. Therefore, as already indicated in the introduction, we use the example of the media industry to explain the interplay of the mechanisms of recombinant digital innovation in service systems and their drivers.

The multiple case study approach allows us to analyze each case per se and derive individual insights, as well as to compare these insights to develop generalizable propositions about the interplay of recombinant mechanisms in the context of digital service systems. Therefore, we selected our cases by applying a criterion sampling logic at first and subsequently a theoretical replication logic to enhance external validity [30]. Through this process, we identified three cases, which are referred to as RaTeCo, TeleCo, and NewsCo. We anonymized the organizations' names due to reasons of confidentiality.

As illustrated in Table 1, all three cases originate from different types of media. RaTeCo is rooted in broadcasting via radio and television, while TeleCo originally solely concentrated on television. Moreover, NewsCo is a newspaper publisher. All three cases have in common that they stretched out to the internet and its various possibilities for distribution as a new media channel. In all cases, the emergence of DigTech and the increasing speed of the internet led to intensified efforts within these companies to migrate their original services to online channels, which will be further outlined in the individual case descriptions. Beyond that, we used their respective content focus as an additional criterion to avoid unilateral results due to a bias towards informational or entertaining formats. Moreover, we additionally used the revenue model, total revenue, and the number of employees for the case selection to acquire potentially heterogeneous data sources that enhance the external validity of our study.

Table 1. Description of case sites

\begin{tabular}{|l|l|l|l|}
\hline Case & RaTeCo & TeleCo & NewsCo \\
\hline Media type & Radio, TV & TV & Newspaper \\
\hline $\begin{array}{l}\text { Content } \\
\text { focus }\end{array}$ & $\begin{array}{l}\text { Local } \\
\text { contents }\end{array}$ & Entertainment & News \\
\hline $\begin{array}{l}\text { Main source } \\
\text { for revenue }\end{array}$ & $\begin{array}{l}\text { License } \\
\text { fees }\end{array}$ & Advertising & Retails \\
\hline $\begin{array}{l}\text { Revenue in \$ } \\
\text { (2019) }\end{array}$ & 1.35 bn & 5.06 bn & 0.29 bn \\
\hline $\begin{array}{l}\text { Employees } \\
(\mathbf{2 0 1 9 )}\end{array}$ & $>3,100$ & $>7,300$ & $>740$ \\
\hline
\end{tabular}

\subsection{Data collection and analysis}

Following Miles et al. [31], the data for our case study primarily focused on semi-structured interviews, supplemented with secondary data from internal documents and presentations, annual reports, and news articles about each company. Between July and October 2020, we conducted ten interviews per case. The interviewees were selected with attention to their professional focus and their hierarchical position within the organization. We interviewed employees from middle- and top-management, that were part of digital innovation processes. Secondary data was collected between January 2019 and March 2021.

All interviews were conducted with the help of a semi-structured interview guideline with open-ended questions. Due to the current Covid-19 pandemic, we interviewed all employees via an online videoconferencing tool. On average, the interviews lasted 
between 30 and 60 minutes. Our guideline followed the conceptual framework introduced before and contained five sections. At first, the interviewees were asked to introduce themselves and their responsibilities within their organization. Secondly, we asked for their individual understanding of recombining DigTech in service systems. At third, we asked for concrete projects where they applied mechanisms for recombination in their organization. Afterward, they were asked to name drivers in- and outside of their company that influenced the recombination of resources in their respective service systems. The interviews ended with an open assessment about the innovation activities of the organization and the opportunity to add further issues that were not addressed earlier. All interviews were recorded digitally and transcribed verbatim. Moreover, we supplemented them with field notes that were created during and subsequent to the interviews. The collected data was stored, coded, and analyzed in a database in ATLAS.ti.

For our data analysis, we performed two cycles of coding [31]. At first, we assigned descriptive codes to our collected data to inductively locate and describe general elements where recombinant mechanisms and their drivers occurred in the organizations' innovation projects. Afterward, we deductively clustered the codes of the first cycle based on our conceptual framework to identify relationships between established digital service solution (EDSS), recombined digital services solutions (RDSS), dissociation mechanisms (DIS), association mechanisms (ASO), addition mechanisms (ADD), and their drivers (DR). Two researchers independently coded the data and afterward jointly derived the three cases based on the mutually condensed results. We exemplified the coding scheme in Table 2.

Table 2. Illustration of the coding scheme

\begin{tabular}{|l|l|l|}
\hline Quote & $\begin{array}{l}\text { Element } \\
\left(1^{\text {st }} \text { cycle }\right)\end{array}$ & $\begin{array}{l}\text { Clusters } \\
\left(2^{\text {nd }} \text { cycle }\right)\end{array}$ \\
\hline $\begin{array}{l}\text { "We divide our } \\
\text { standard services into } \\
\text { many distinct micro } \\
\text { services to use them } \\
\text { independently." }\end{array}$ & $\begin{array}{l}\text { Splitting up } \\
\text { established } \\
\text { solutions into } \\
\text { resources }\end{array}$ & EDSS, DIS \\
\hline $\begin{array}{l}\text { "The more expensive a } \\
\text { new project would be, } \\
\text { the more we test its } \\
\text { components within the } \\
\text { services that are already } \\
\text { in use" }\end{array}$ & $\begin{array}{l}\text { Risk reduction } \\
\text { through } \\
\text { additive } \\
\text { recombination }\end{array}$ & $\begin{array}{l}\text { DR, ADD, } \\
\text { RDSS }\end{array}$ \\
\hline $\begin{array}{l}\text { "We stored our contents } \\
\text { digitally independent of } \\
\text { their original formats } \\
\text { before we were able to } \\
\text { reuse them in other } \\
\text { channels." }\end{array}$ & $\begin{array}{l}\text { Splitting up } \\
\text { established } \\
\text { solutions to } \\
\text { reuse them } \\
\text { within other } \\
\text { solutions }\end{array}$ & $\begin{array}{l}\text { EDSS, ASO, } \\
\text { RDSS }\end{array}$ \\
\hline
\end{tabular}

\section{Results}

In the following, the results from the three cases are presented in two steps. At first, we use case descriptions to explain the setting of digital recombination activities within the analyzed organizations. Secondly, we summarize how the recombination mechanisms are applied and which drivers occurred in comparison of the three cases. When citing interviewees directly in this section, we use the anonymized name of the case (i.e. $\mathrm{RaTeCo}$, TeleCo, and NewsCo) and add a number to it to indicate the interviewee.

\subsection{Case descriptions}

RaTeCo is part of a public communication network in Germany and one of the largest radio and TV stations in Europe. The pervasion of DigTech in the media industry forced RaTeCo to develop vigorously new digital services for their audience that widely address various channels. Therefore, they focused on the development of their own on-demand platform that offered users the possibility to listen and watch their contents which were originally presented on their linear channels only. From an organizational perspective, the implementation of online channels leads to structural changes within the company that aimed to enable the compatibility of the offered services with regard to the provided channels. Originally, their departments were clustered based on a channel structure. This means that the radio-, the TV-, and the online department created their own contents and worked largely autonomously. However, after the adjustment of the organizational structure, the departments were detached from specific media types and channels to foster collaborative innovation between the content types. For example, TV shows about a certain topic were no longer planned with a strict focus on being aired solely on linear TV. In fact, they are now being produced with the aim to reuse these contents in other channels of RaTeCo, which can be seen as a recombination of diverse services, such as their online library, their YouTube channel, their SmartTV application, and their podcast channels.

TeleCo is one of the biggest and earliest private TV broadcasting stations in Germany. The influence of DigTech originally led this organization to acquire new subsidiaries that are active in other business fields such as e-commerce and technology consulting. However, especially in the last five years, they noticed that turning away from their core business (i.e. producing and distributing contents) lead to decreasing market shares in their main audience. Consequently, the broadcaster decided to refocus on the media business area by recombining its individual channels with the aim to enhance the value propositions offered to its audience. 
From an organizational perspective, TeleCo wanted to support their core competency (i.e. content distribution) with further digital expertise to enhance the interconnectedness of their respective service system. Therefore, they established a dedicated department that solely focused on digital media and its opportunities. This department had two central missions: On the one hand, its members initiated projects to create new digital service solutions (e.g. introducing a new quiz app based on one of their most profitable TV programs). On the other hand, they served as ambassadors for digital innovation in other project teams.

NewsCo is a newspaper publisher that has been operating in Germany for over 60 years. It is popular for its focus on high-quality news content and politically balanced reporting. Moreover, its worldwide network of correspondents is one of the biggest internationally, which also has an impact on the topicality of its press coverages. Due to a constantly decreasing circulation and declining numbers of subscribers of the printed version of its newspaper since 2000 , the publisher was forced to take action and realign its business by implementing new digital services. Therefore, they focused on setting up new channels such as a news website, diverse news apps, and an informative daily podcast to distribute their content. During the implementation of these new online channels, their editorial team organizationally remained unchanged and continued to focus on producing informational contents. Therefore, they additionally formed a technical department that focuses on maintaining their online channels. However, this department is not deliberately in charge of innovation processes in this service system.

\subsection{Case analysis}

By comparing the three described cases, it became salient that mechanisms of recombinant digital innovation in service systems are a predominant approach in the media industry. The aim of the application of the mechanisms is to enhance the interconnectedness of the resources of established digital service solutions (EDSS) to develop recombined digital service solutions (RDSS) that offer new value propositions to their customers. The application of the mechanisms is influenced by diverse drivers (DR). Therefore, we will initially address how dissociative, associative, and additive mechanisms of recombinant digital innovation were applied in the analyzed organizations. Afterward, we will outline which drivers were pivotal for each mechanism.

Dissociation mechanisms (DIS): All three organizations stated that dissociation mechanisms play a central role in their innovation processes. They use dissociation to compartmentalize EDSSs into their resources. For example, contents can be "produced for a Youtube-Channel, however, they are also used in the news show in linear TV" (RaTeCo-1). This is enabled by splitting most of the produced contents into several delimited video/audio clips, which is also referred to as clipping. Due to this clipping into content components, the flexibility to recombine them with each other and with diverse channels increases. Consequently, the organizations enhance the interconnectedness of their modules by decoupling them from their original EDSS. For RaTeCo and TeleCo, this is enabled with the help of an integrated content- and service-management system (C-/SMS). This system serves as a database as it stores information about every piece of produced content and how these contents can be recombined with other services. This information not only contains details about the topics of the contents but also about their compatibility with diverse channels. In general, these systems help them to sense opportunities for the association of recombinable modules. They provide an overview of which contents already exist and how they might be reutilized with different channels to develop new RDSS. Consequently, this technical management infrastructure helps them to "produce differently than before" (RaTeCo-6). In return, contents are produced without focusing on a certain service but with the aim to be modular in order to be reused with diverse types of channels. In essence, this builds up a system of services that can be potentially offered. Interestingly, NewsCo did not implement such a modular infrastructure so far. However, their contents need to be distributed to their audience as fast and far-reaching as possible, which is why the organization plans to introduce a similar system in the near future.

Drivers for dissociation: The dissociation of EDSS is mostly influenced by factors originating from "the market, technological developments and in a best-case scenario the user themselves" (NewsCo-4). These drivers can be subsumed as external facilitators that inspire organizations to analyze their EDSS and compartmentalize them into DigTech modules, which can be recombined subsequently. From a market perspective, these external drivers are impulses that stem from the competitive environment of the organization. These impulses enhance the urgency to develop a new digital service solution, which is possible via recombination: Applications of dissociative mechanisms "are reactions to some effect or occurrence, either on the direct market [...] or on an adjacent market" (TeleCo-7). This influence of external drivers on dissociative mechanisms of recombinant digital innovation is exemplified by NewsCo-5 as follows: "When [a competitor] releases a new app, we test it and see if it is a good idea. Maybe we develop something similar then." Dissociation is especially important here 
as it enables organizations to directly respond with the help of DigTech that are already established internally.

Besides that, customer demands can also serve as external impulses for dissociating EDSS in service systems, as this helps to "precisely meet customer needs." (RaTeCo-8). To achieve this goal, RaTeCo has its own data analytics team that searches for trends. For example, this team identified the need for an own ondemand platform by analyzing changes in the watching patterns of the audience of their linear channels. This observation induced the organization to split its linear contents up into modules that can be reused on diverse channels such as its own media library. This made it possible to watch programs that were already broadcasted on radio or TV at a later point in time. Consequently, it is possible to increase the offered value proposition by modularizing EDSS because of customer demands. Another example for user-induced dissociation of an established digital service solution is the Covid-19-Newsletter service of NewsCo: Due to the actuality of the pandemic, their audience requested daily information about the current statistics of the pandemic. Instead of developing such a service from scratch, they modularized all available contents about the pandemic that were offered via diverse other services (i.e. EDSSs) and thereby made them reusable for other channels. This enabled the distribution of statistical information about the pandemic simultaneously. In sum, dissociation mechanisms are applied to separate the resources of EDSSs and prepare them to be recombined with other elements to generate a new RDSS, which is facilitated by external drivers that stem from competitors or users.

Association mechanisms (ASO): Association mechanisms are applied to develop new RDSS. As a basis for associative recombination, the organizations assess which digital modules are recombinable within their service system and how they can be connected with each other. To apply this mechanism, it is important that the DigTech that are already part of an EDSS can easily be adapted to other contexts. Thereby, it became salient that associative mechanisms specifically focus on the recombination of DigTech that are already modularized internally. One example of such a recombined digital service solution is the daily news app of RaTeCo. The goal of this app was that their breaking news reports should not only be available via their website but also as a push notification on smartphones. Hence, they connected the breaking news section of their website with their news app. Thereby, they enabled that once these sections are updated on one of the two channels, the other channel automatically updates as well. Due to this association of the DigTech of two EDSS, they generated a new value proposition in form of a joint RDSS. This type of reusing content modules by associating them with the technologies of other EDSS in the service system is also applied by TeleCo: "If we broadcast content on TV [...] we additionally distribute it via Facebook, Youtube, and others, and we cut out short consumable pieces and distribute them via IGTV for example." (TeleCo-2). Hence, it can be implied that the application of associative mechanisms is dependent on the modularization and therefore recombinability of the resources within a service system. From this perspective, it becomes evident that dissociative mechanisms serve as an antecedent for association.

Drivers for association: As indicated before, the modularization of the DigTech of EDSS through dissociation leads to the emergence of recombinable digital modules that can be reconfigured. By recombining these modules with each other, new RDSSs can be generated that offer an additional value proposition. Consequently, the association mechanism relies mostly on the reutilization of DigTech that are already part of a service system. The central driver that facilitates such associative mechanisms of recombinant digital innovation is the internal driver to reduce costs while simultaneously develop valuable innovative solutions. Therefore, cost reduction can be introduced as the central internal driver to conduct associative recombination. In terms of the media industry, this means that the modularization of an EDSS, which is enabled by DIS, serves as a prerequisite to efficiently apply ASO, which generates new RDSS. This in return enhances the flexibility of organizations as it enables them to innovate by solely associating existing resources within their service system. This is especially important in the media industry, as the value of their service solutions is to a certain degree dependent on its topicality. Recombination is especially useful here as it bypasses the cost-intensive assessment and adoption of new digital distribution technologies.

Addition mechanisms (ADD): Addition mechanisms were especially applied when the organizations wanted to test how new technologies could be used within their current service systems. Therefore, they added a new DigTech to the configuration of an EDSS. Thereby they expanded the value proposition of this service by developing a RDSS. An example of such a technology is the possibility to use interface overlays in linear TV in real-time. TeleCo additively recombined this DigTech for live polling in their program. Hence, they developed a survey function in their add-on application for mobile devices. By recombining these two services/channels (i.e. linear TV and polling in the mobile app) they were able to show the audience's reaction to a certain topic while it is currently being aired on TV.

Drivers for addition: As outlined before, addition mechanisms of recombinant digital innovation help to pretest how individual (new) DigTech can fit into the 
service system of an organization. This is mostly motivated by the fact that if organizations would "build something completely new, what no other competitor has, [they would] have no references for comparison" (NewsCo-6). Consequently, additive mechanisms are mostly motivated because of an internal driver to reduce risk. By adding a DigTech to an EDSS a new RDSS is generated which helps the organizations to analyze the potential of this technology for their respective service system. Therefore, risk reduction is introduced as the central internal driver for additive recombination.

\section{Discussion}

The results of our study provide insights into the interplay between the three mechanisms of recombinant digital innovation, namely dissociation, association, and addition, as well as into the drivers that influence the individual application of these three mechanisms. Based on our findings, we therefore suggest the following three propositions:

Proposition 1: An established digital solution can be compartmentalized into recombinable modules through dissociation and afterward reconfigured through association of these modules to generate a recombined digital service solution.

An explanation for the intense dependency between dissociation and association can be found in the properties that digital solutions imply. They are composed of diverse DigTech, which jointly form a usable artifact that provides a value proposition. In return, an isolated DigTech cannot form a usable service solution solely by itself [5]. Due to this need for the interconnectedness between digital resources, dissociation cannot lead to the emergence of a recombined digital service solution by itself, as it aims at splitting up the involved DigTech in an established solution to utilize them independently. Therefore, dissociation can be seen as the central prerequisite for association, as it helps to modularize all digital resources established in a service system and therefore makes them recombinable (i.e. associable). As a result, association mechanisms process these resources by reconfiguring them into RDSS.

Proposition 2: If an established digital service solution is supplemented with a (new) DigTech, this generates a recombined digital service solution through addition.

As outlined before, addition mechanisms aim at omitting the risks of implementing completely new and untested service solutions. Thereby they abstract new individual resources (i.e. DigTech) that are not yet implemented in a service system to recombine them with an established solution [22]. This enables a lowrisk framework that helps to identify potential options for DigTech that can potentially be added to a service system to effectively generate new recombined digital service solutions.

Proposition 3: The application of mechanisms of recombinant digital innovation is enhanced by different external and internal drivers.

While the application of dissociative mechanisms is mainly triggered from outside of the organization through competitors and users. The application of associative mechanisms is mainly facilitated from inside the organization through the driver to reduce costs. Moreover, the application of additive mechanisms is also mainly facilitated from inside the organization through the driver to reduce risks.

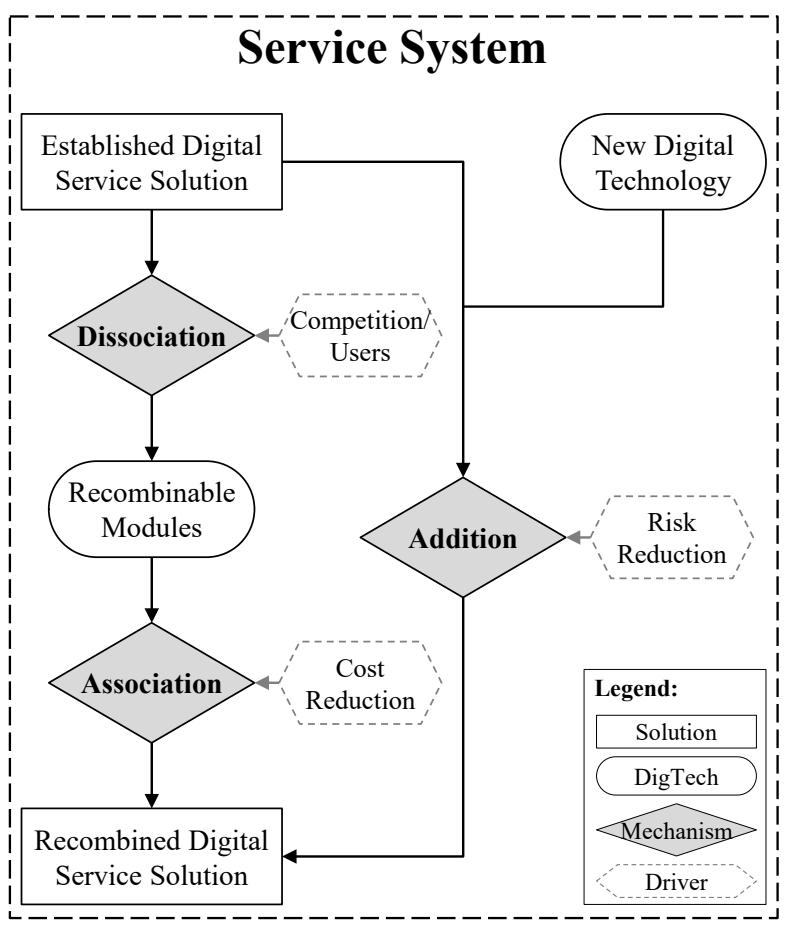

Figure 2. Concatenation of recombinant digital innovation in service systems

We summarized our findings in Figure 2, by developing a conceptual model for the concatenation of mechanisms for recombinant digital innovation in service systems, which helps to visualize the relationship of the three propositions: Within a service system, a set of established digital service solutions exists. The application of mechanisms of recombinant digital innovation helps to design recombined digital service solutions that provide new value propositions. This engineering process can follow two paths: On the one hand, the dissociation of established digital service solutions leads to the generation of recombinable digital modules, which is mostly triggered by influences from 
competitors and users. The drive to generate new value propositions while reducing costs then leads to the association of these modules, which results in the generation of recombined digital service solutions. On the other hand, such solutions can also be generated through the addition of a new DigTech to an established digital service solution in a service system, which helps the organization to manage risks incorporated by the implementation of unknown resources.

\section{Contribution, limitations, and conclusion}

By applying the concept of the mechanisms of recombination to digital innovation in media organizations, we contribute to literature from service system theory and digital innovation $[3,5,10,11,17]$ in twofold ways:

First, we answer research question 1 by showing that the application of dissociation, association, and addition mechanisms in service development is interdependent and can follow two pathways to generate new value propositions in form of recombined digital service solutions. On the one hand, established solutions can be dissociated into recombinable DigTech modules that can be associated afterward to form a recombined digital service solution. On the other hand, such recombined solutions can be generated by adding new DigTech to established digital service solutions. Second, we answer research question 2 by discovering that the distinct recombination mechanisms are facilitated by internal and external drivers, which differ for the application of each of the three mechanisms.

By using the example of the media industry, our findings give first empirical evidence for the concatenation of recombinant mechanisms and therefore shed light on the applicability of the concept of recombination in the context of digital innovation [3, 5, 11]. Moreover, our developed conceptual model expands the valuable concept of Beverungen et al. [10] as it shows that recombination mechanisms are rather applied in a mutually supplementary than substitutional manner. Against this background, we develop "explanations of digital innovation building on the specifics of digital technologies" [3, p. 110] in the context of service systems. Hence, our findings shed light on the importance of digital services and their interconnectedness by focusing on distinct DigTech as their central resource [17]. Therefore, our results can serve as a starting point for further empirical research on (digital) service systems in diverse industries in general as well as the development of recombined digital service solutions in the media industry in particular [16].

From a practical perspective, managers should generally recognize recombinant digital innovation as a promising approach to generate value propositions in form of recombined digital service solutions with the opportunity to reduce risks that emerge through the possibility of failing implementation processes and costs that occur due to the acquisition of resources, which might in fact not be necessary. Therefore, our developed conceptual model can serve as a useful framework for organizational decision-makers to understand the concatenation of the mechanisms of recombinant digital innovation, which helps them to decide for the individual application of the mechanisms in their respective organization in coordination with their strategic focus. By highlighting the interdependency of the three mechanisms and giving examples from the media industry, our work can serve as a useful blueprint to enhance the application of mechanisms for recombination in manifold settings.

However, our study also has a few limitations. First, we only analyzed companies from the media industry. Hence, it would be reasonable to imply that our results could behave differently for organizations with a less intense focus on the development of services. Although we used a criterion sampling logic for our case selection, we suggest conducting further case research about recombination mechanisms in different contexts. Moreover, the media industry is highly dependent on the distribution of consumable contents, which certainly influences the degree of possible modularity within an organizational service system. However, we argue that due to the pervasive digitalization nearly every type of organization offers at least some type of content to its customers. For example, our results could also be transferred to the automotive industry where contents such as map data and information about driving performance can serve as input for the recombination of DigTech to generate new recombined digital service solutions. Second, the results of this study only focus on a technical design recombination perspective. However, innovation decisions are also often influenced by political and strategic decisions as well as individual organizational capabilities [e.g. 32]. Consequently, empirical research on digital recombinant innovation in service systems from other research lenses should also provide promising insights. Third, the exploratory identified drivers for dissociation, association, and addition need further empirical examinations to preclude potential overlaps between them.

Overall, our study is an initial step towards a better understanding of the concrete application of digital recombination. By using the example of the media industry, we shed light on the interplay between the mechanisms of recombinant innovation in service systems and their potential drivers. We hope that this study gives an impetus for further research about recombination in general and digital recombinant innovation of service solutions in particular. 


\section{References}

[1] J.A. Schumpeter, Theory of Economic Development, Routledge, New York, USA, 1934.

[2] F. Gallouj, and O. Weinstein, "Innovation in Services", Research Policy, 26(4-5), 1997, pp. 537-556.

[3] J. Holmström, "Recombination in Digital Innovation: Challenges, Opportunities, and the Importance of a Theoretical Framework", Information and Organization, 28(2), 2018, pp. 107-110.

[4] G. Vial, "Understanding Digital Transformation: A Review and a Research Agenda", The Journal of Strategic Information Systems, 28(2), 2019, pp. 118-144.

[5] O. Henfridsson, J. Nandhakumar, H. Scarbrough, and N. Panourgias, "Recombination in the Open-Ended Value Landscape of Digital Innovation", Information and Organization, 28(2), 2018, pp. 89-100.

[6] Y. Yoo, R.J. Boland Jr, K. Lyytinen, and A. Majchrzak, "Organizing for Innovation in the Digitized World", Organization Science, 23(5), 2012, pp. 1398-1408.

[7] Y. Yoo, O. Henfridsson, and K. Lyytinen, "Research Commentary - the New Organizing Logic of Digital Innovation: An Agenda for Information Systems Research", Information Systems Research, 21(4), 2010, pp. 724-735.

[8] A. Benlian, W. Kettinger, A. Sunyaev, and T. Winkler, "The Transformative Value of Cloud Computing: A Decoupling, Platformization, and Recombination Theoretical Framework", Journal of Management Information Systems, 35(3), 2018, pp. 719-739.

[9] T.J. Saldanha, A. Sahaym, S. Mithas, M.G. AndradeRojas, A. Kathuria, and H.-H. Lee, "Turning Liabilities of Global Operations into Assets: IT-Enabled Social Integration Capacity and Exploratory Innovation", Information Systems Research, 31(2), 2020, pp. 361-382.

[10] D. Beverungen, H. Lüttenberg, and V. Wolf, "Recombinant Service Systems Engineering", Business and Information Systems Engineering, 60(5), 2018, pp. 377-391.

[11] A. Hund, "Recombination in Times of Pervasive Digitalization: A Review", ICIS 2020 Proceedings, 2020

[12] L. Hylving, and U. Schultze, "Accomplishing the Layered Modular Architecture in Digital Innovation: The Case of the Car's Driver Information Module", The Journal of Strategic Information Systems, 29(3), 2020, pp. 1-23.

[13] O. Lundberg, J. Sandberg, and D. Nylén, "Cycles of Innovation and Alignment in Digital Transformation: Investigating the Dynamics of Resource Recombination in a Construction Firm", HICSS 2020 Proceedings, 2020

[14] S. Nambisan, K. Lyytinen, A. Majchrzak, and M. Song, "Digital Innovation Management: Reinventing Innovation Management Research in a Digital World", MIS Quarterly, 41(1), 2017, pp. 223-238.

[15] R. Alt, and C. Militzer-Horstmann, "Electronic Markets on the Media Industry", Electronic Markets, 27(1), 2017,

[16] T. Hess, and I. Constantiou, "Introduction to the Special Issue on "Digitalization and the Media Industry", Electronic Markets, 28(1), 2018, pp. 77-78.

[17] S. Knop, E. Galipoglu, A. Lubarski, and J. Poeppelbuss, "Quo Innovadis? The who, the what and the how of Research at the Intersection of ICT and Service Innovation", ICIS 2017 Proceedings, 2017
[18] P.P. Maglio, and J. Spohrer, "Fundamentals of Service Science", Journal of the Academy of Marketing Science, 36(1), 2008, pp. 18-20.

[19] J. Spohrer, P.P. Maglio, J. Bailey, and D. Gruhl, "Steps Toward a Science of Service Systems", Computer, 40(1), 2007, pp. 71-77.

[20] F. Wiesböck, and T. Hess, "Digital Innovations", Electronic Markets, 30(1), 2020, pp. 75-86.

[21] L. Fleming, "Recombinant Uncertainty in Technological Search", Management Science, 47(1), 2001, pp. 117-132.

[22] H.M.L. Waltermann, and T. Hess, "Competitive Recombination of Digital Technologies in the TV-Media Industry", AMCIS 2020 Proceedings, 2020

[23] R.K. Yin, Case Study Research: Design and Method, SAGE Publications, 5th, Thousand Oaks, 2013.

[24] G. Paré, "Investigating Information Systems With Positivist Case Research", Communications of the AIS, 13(1), 2004, pp. 233-265.

[25] L. Dubé, and G. Paré, "Rigor in Information Systems Positivist Case Research: Current Practices, Trends, and Recommendations", MIS Quarterly, 27(4), 2003, pp. 597-636. [26] M.D. Myers, Qualitative Research in Business and Management, SAGE Publications, London, UK, 2009.

[27] S.L. Vargo, and R.F. Lusch, "Evolving to a New Dominant Logic for Marketing", Journal of Marketing, 68(1), 2004, pp. 1-17.

[28] T. Hess, "What Is a Media Company? A Reconceptualization for the Online World", International Journal on Media Management, 16(1), 2014, pp. 3-8.

[29] I. Benbasat, D.K. Goldstein, and M. Mead, "The Case Research Strategy in Studies of Information Systems", MIS Quarterly, 11(3), 1987, pp. 369-386.

[30] M.Q. Patton, Qualitative Research \& Evaluation, SAGE Publications, 3rd, Thousand Oaks, USA, 2002.

[31] M. Miles, A.M. Huberman, and J. Saldana, Qualitative Data Analysis - a Methods Sourcebook, SAGE Publications, Thousand Oaks, USA, 2013.

[32] F. Wiesböck, T. Hess, and J. Spanjol, "The dual role of IT capabilities in the development of digital products and services", Information \& Management, 57(8), 2020, pp. 1-48. 